\title{
Birds of the Reserva Biológica do Mato Grande and surroundings, Rio Grande do Sul, Brazil
}

\author{
Jeferson Vizentin-Bugoni ${ }^{1,2,3 *}$, Fernando Jacobs ${ }^{3}$, Marco Antônio Afonso Coimbra ${ }^{4}$ and \\ Rafael Antunes Dias ${ }^{3}$
}

1 Pós-graduação em Ecologia, Instituto de Biologia, Universidade Estadual de Campinas, CEP 13083-865, Campinas, SP, Brazil

2 Center for Macroecology, Evolution and Climate, University of Copenhagen, Universitetsparken 15, 2100 Copenhagen, Denmark

3 Laboratório de Ecologia de Aves e Mamíferos, Departamento de Ecologia, Zoologia e Genética, Instituto de Biologia, Universidade Federal de Pelotas, CP 354, CEP 96010-900, Pelotas, RS, Brazil

4 Núcleo de Reabilitação da Fauna Silvestre e Centro de Triagem de Animais Silvestres, Instituto de Biologia, Universidade Federal de Pelotas, CEP 96010-900, Pelotas, RS, Brazil

* Corresponding author. E-mail: jbugoni@yahoo.com.br

\begin{abstract}
The Reserva Biológica do Mato Grande encompasses 5,161 hectares of wetlands, restinga forests and grasslands in southern Brazil. Aiming to assemble a list of bird species occurring in the reserve, we carried out 21 monthly expeditions from July 2007 to March 2009 and an additional visit on October 2014, totaling 341 hours of sampling. We additionally searched for records in online databases and museums. In total, 211 species of birds were found, compared to 223.83 $(\mathrm{SD}=3.88)$ and $214.68(\mathrm{SD}=4.71)$ species respectively predicted through Jackknife 2 and Chao 2 estimations. Plegadis chihi was the most abundant bird roosting in the reserve. The area is important for the conservation of Circus cinereus, Spartonoica maluroides, Limnoctites rectirostris and Sporophila palustris, which are considered threatened or near-threatened in state, national and/ or global levels. We emphasize the urgent need of implementing the Reserva Biológica do Mato Grande in order to conserve the regional avifauna.
\end{abstract}

Key words: wetlands, restinga forests, grasslands, conservation, protected areas

\section{INTRODUCTION}

Wetlands exhibit high levels of primary productivity and are crucial for the maintenance of the life cycles of various aquatic and terrestrial organisms (EPA 2014). Apart the intrinsic importance for the maintenance of biodiversity, wetlands have considerable economic value in terms of ecosystem services such as fishery resources, usage as agricultural and recreational purposes, and flood mitigation, among others (Barbier et al. 1997). However, the maintenance of the functions and services of wetlands depends on the integrity of ecological processes (e.g., decay of organic matter by microorganisms, exchange of matter and nutrients driven by migratory organisms such as fishes and birds associated with water movements) within these areas (Barbier et al. 1997; Keddy 2000). Wetlands have suffered high rates of habitat loss and degradation and are considered one of the most threatened ecosystems in the world (Maltby 1991). Major threats to wetlands include drainage, filling, conversion into urban and agricultural areas, pollution, and overexploitation of resources. These impacts modify wetland biodiversity (Weller 1999), resulting in the loss of the ecological processes that are important for the dynamics and stability of these ecosystems (Barbier et al. 1997; Keddy 2000).

The establishment of protected areas is one of the main strategies to prevent biodiversity loss in wetlands (Keddy 2000). However, to fulfill their conservation goals, protected areas must be properly implemented and have their biodiversity known (Furness and Greenwood 1993). Harboring 3,441 wetlands, the state of Rio Grande do Sul, in southern Brazil, is one of the regions with the largest number of these ecosystems in South America (Maltchik et al. 2003). These wetlands are potentially important for birds, not only because they provide foraging, roosting and breeding habitat for resident species, but also for their role as stopover sites or wintering areas for several migrants of both Nearctic and Neotropical origins (Belton 1994; Guadagnin et al. 2005; Bencke et al. 2007). However, bird inventories are available only for a few wetlands of Rio Grande do Sul (Mähler-Jr. et al. 1996; Accordi and Barcellos 2006; Accordi and Hartz 2006; Bencke et al. 2007; Harrison et al. 2013). Lack of adequate information on bird species 
inhabiting wetlands greatly limits the development and establishment of efficient conservation strategies.

Located in the coastal region of southern Brazil, the Reserva Biológica do Mato Grande is a 5,161 ha protected area encompassing various wetland types, restinga forests and grasslands. Despite being situated in the Canal São Gonçalo "BirdLife International Important Bird Area" (Bencke et al. 2006), a region also highlighted as an "area of extreme importance for the conservation, sustainable use and benefit sharing of the Brazilian biodiversity" (MMA 2007), the avifauna of the reserve remains poorly known. The first ornithological study in the area was carried out by Emil Kaempfer, which collected birds around the village of Santa Isabel in 1931 (Naumburg 1935). Later, in the 1970s, William Belton observed, tape-recorded and collected birds in this same locality, as well as in farms that include portions of the reserve within their limits (Belton 1994). Despite being visited by two of the most active bird collectors in the ornithological history of Rio Grande do Sul, a list of birds recorded in the reserve and surrounding area does not exist.

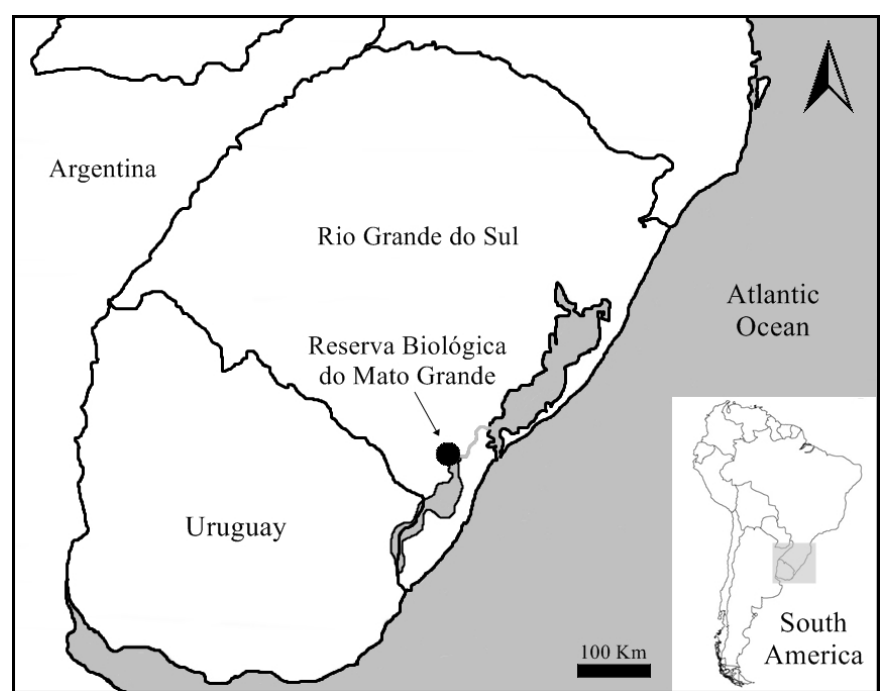

Figure 1. Map showing the location of the Reserva Biológica do Mato Grande, state of Rio Grande do Sul, Brazil.

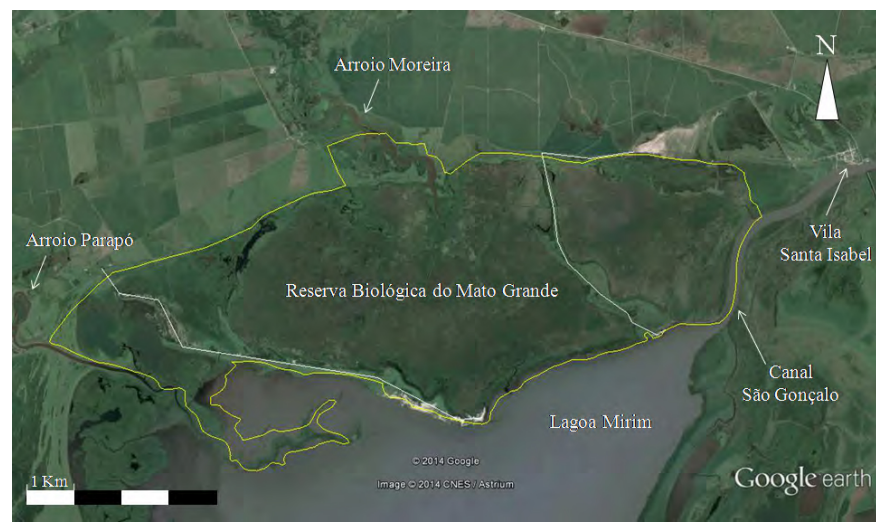

Figure 2. Limits (yellow) of the Reserva Biológica do Mato Grande, Brazil. The white lines indicate bird sampling transects in the eastern (right) and western (left) sectors of the reserve. Source: Google Earth ${ }^{\mathrm{Tm}}$.
Here, we aimed to list bird species occurring in the Reserva Biológica do Mato Grande and surroundings. We surveyed birds in the field and searched for additional records in museums and virtual repositories of voice recordings and photographs. We followed guidelines to adequately list and document species in avian inventories (Bencke et al. 2010; Lees et al. 2014). We also provide data of abundance for species of conservation concern and for species congregating in roosts within the reserve. Based on our findings, we discuss the importance of this protected area for the conservation of endangered, endemic and/or wetland birds in the region.

\section{MATERIALS AND METHODS \\ Study area}

The Reserva Biológica do Mato Grande (RBMG) $\left(32^{\circ} \mathrm{O} 8^{\prime}\right.$ $\left.\mathrm{S}, 52^{\circ} 4 \mathrm{O}^{\prime} \mathrm{W}\right)$ is a state-administrated biological reserve located in Arroio Grande municipality, southern Rio Grande do Sul, Brazil (Figure 1). The boundaries of the reserve are the Lagoa Mirim to the south, the Canal São Gonçalo to the east, the Arroio Parapó to the west, and grasslands and rice paddies situated on higher ground to the north (Figure 2). The decree establishing this protected area dates from 1975 (State Decree 23.798/75). Although a manager has been recently designated, full implementation of the reserve, including land use and tenure, remains pending.

Vegetation in the RBMG is composed largely by pioneer species established in geologically recent areas such as the Coastal Plain of Rio Grande do Sul (IBGE 1986) (Figure 3). Most of the reserve is comprised by freshwater marshes dominated by various species of emergent (Scirpus spp., Schoenoplectus californicus (C.A. Mey.) Palla., Zizaniopsis sp.) and floating (Pistia, Nymphoides, Eichornia, Salvinia) aquatic macrophytes. Sarandi (Phyllanthus sellowianus (Klotzsch) Müll.Arg., Sebastiania schottiana (Müll. Arg.)) bushes are also common. Seasonally flooded grasslands are found marginally to the marshes. Stands of Panicum prionitis Nees and Eryngium pandanifolium Cham. \& Schltdloccur in these areas. Comparatively higher grounds within the reserve are covered with sandy grasslands and restinga forests. The arborescent stratum of the latter is comprised mostly by Erythrina crista-galli L., Ficus spp, and Syagrus romanzoffiana (Cham.) Glassman, while the lower stratum is dominated by the shrubby Psychotria spp., Justicia brasiliana Roth and Calliandra tweedii Benth. Patches of Bromelia antiacantha Bertol. are also common in forest edges. Short segments (up to $1 \mathrm{~km}$ ) of sandy or muddy beaches are found along the margins of the Lagoa Mirim. Cattle roam free inside the reserve and illegal hunting, fishing and occasional bird trapping also occur. Areas adjacent to the reserve are used for irrigated rice cultivation. Small patches of eucalyptus and restinga forests are found in these areas, as well as a 
few grassland and wetland remnants.

Climate in the region is sub-humid (IBGE 1986). In the neighboring municipality of Capão do Leão, mean annual temperature is $17.8^{\circ} \mathrm{C}$, mean annual rainfall is $1.366 \mathrm{~mm}$ and mean relative humidity is $80.7 \%$ (Estação Agroclimatológica de Pelotas 2014). The annual water balance in the region is as follows: deficit from December to March, reposition in April and May, surplus from June to September, and withdrawal in October and November (Klein 1998). Water level in wetlands is consequently lower in late summer and early autumn and higher in the winter and early spring.

\section{Data acquisition and analysis}

Between July 2007 and March 2009 we carried out 21 monthly expeditions to the study area. An additional expedition was carried out on 23 October 2014. Expeditions lasted 1-2 days each (10-34 h of sampling effort), totaling 341 hours of fieldwork. We surveyed two sectors of the RBMG (Figure 2). The western part of the reserve was visited on 13 occasions. We searched for birds mainly along an $8.5 \mathrm{~km}$ transect, from rice lands bordering the reserve to the restinga forest and sandy/muddy beaches of the Lagoa Mirim. The eastern part of the reserve was visited nine times. We surveyed birds mostly along a
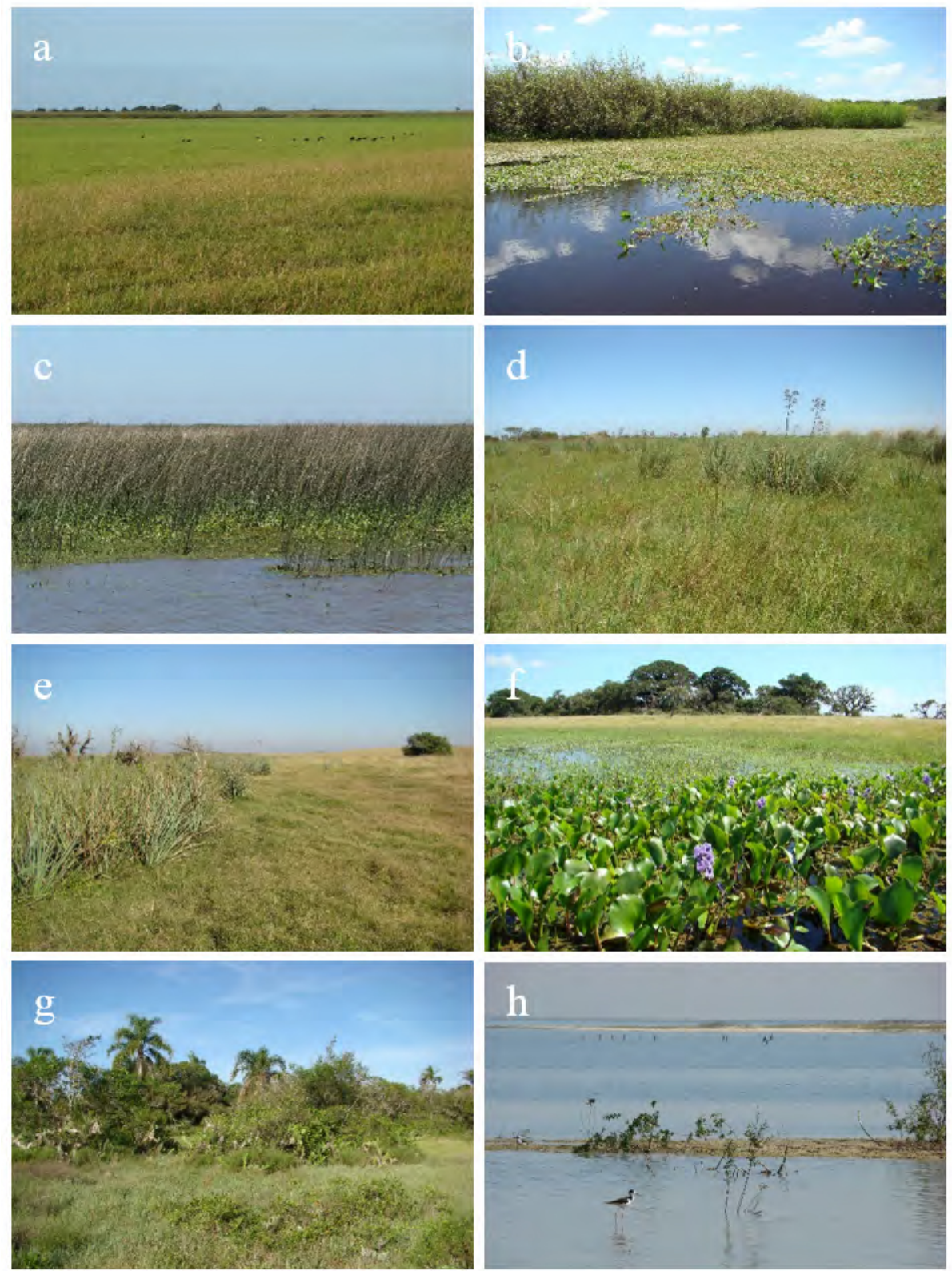

Figure 3. Major habitat features of the Reserva Biológica do Mato Grande, Brazil. (a) Open grassy marsh at the transition with sandy grassland over ancient beach ridge. (b) Sarandi bushes and tall emergent herbaceous plants such as Zizaniopsis sp. (right) cover most of the central sector of the reserve. (c) Stands of Schoenoplectus californicus are also common in marshes. (d) Panicum prionitis tussocks and the spiny Eryngium pandanifolium dominate the upper stratum of seasonally flooded grasslands along the Canal São Gonçalo. (e) An E. pandanifolium (left) patch marking the transition between wetlands and dryer grasslands in higher ground (right) along the northeastern limit of the reserve. (f) Floating aquatic macrophytes in open marsh with restinga forest over beach ridge in the background; large trees are Ficus sp. and Erythrina crista-galli. (g) Syagrus romanzoffiana palms in restinga forest over sandy beach ridge. (h) Muddy and sandy beaches with small sarandi bushes along the Lagoa Mirim. Photographs by RAD, except (c) by JVB. 
$6 \mathrm{~km}$ transect, from rice growing areas adjacent to the reserve to flooded grasslands and the sandy beach at the Lagoa Mirim. Vegetation in both sectors was overall similar, with a slight predominance of open marsh with floating macrophytes in the west, and seasonally flooded grasslands with $P$. prionitis and E. pandanifolium in the east. We ensured that bird surveys covered annual seasons equally in both sectors.

Birds were detected and identified visually with the aid of binoculars and by recording and comparing vocalizations. We played-back voice recordings of secretive species such as marsh-dwelling rails and furnariids to ensure detection. Species detected near the RBMG (up to $5 \mathrm{~km}$ from the limits of the reserve) were included in the list. For species of conservation importance, we also recorded the number of individuals detected each time the species was found. We further counted roosting birds within the reserve. Counts were made from prominent points were individuals flying into the roosting areas could be visualized. Records were documented with photographs (deposited at www.wikiaves. com) and/or digital recordings of their voices (deposited at www.xeno-canto.org). Documented records can be accessed online using the voucher numbers listed in Table 1. We also searched for specimens from the RBMG and vicinities deposited in ornithological collections. We visited the Museu de Ciências Naturais (MCN) of the Fundação Zoobotânica do Rio Grande do Sul, and the Museu de Ciências e Tecnologia (MCT) of the Pontifícia Universidade Católica do Rio Grande do Sul, both located in Porto Alegre, RS, Brazil. We also searched the ORNIS digital database (http://www.ornisnet.org) for specimens housed in North American collections and recordings deposited in the Macaulay Library (http:// macaulaylibrary.org). We only listed specimens whose collection locality could be safely attributed to the RBMG, either by a direct reference to the area in the label or by plotting the geographic coordinates available on the label on a map.

Scientific nomenclature and species sequence follows CBRO (2014). Because the RBMG lies within the area of overlap of the ranges of Cranioleuca obsoleta (Reichenbach, 1853) and Cranioleuca pyrrhophia (Vieillot, 1818) and that hybridization is expected to occur (Belton 1994; Claramunt 2002), we treated all individuals of this genus recorded in arboreal formations of the reserve as Cranioleuca sp. We listed species considered endemic of the Pampas and Atlantic Forest biogeographic provinces according to Bencke et al. (2006). Threat status in state, national and global levels follows Rio Grande do Sul (2014), MMA (2014) and IUCN (2014), respectively. We provided detailed information of our records for species of conservation concern and/or birds with poorly known distributions in Rio Grande do Sul. We considered the record of a given species for the RBMG a distributional novelty within Rio Grande do Sul when the nearest state record provided in Belton (1994), Mähler-Jr. et al. (1996), Dias and Maurício (1998), Maurício and Dias $(1996,2000)$ and Bencke et al. (2003) lies over $100 \mathrm{~km}$ from the limits of the reserve. We also considered distributional novelties species known from less than five localities in southern Rio Grande do Sul (i.e., south of $31^{\circ} \mathrm{S}$ ) according to these same sources.

In order to assess sampling completeness, we used the second-order Jackknife and Chao species richness estimators (Gotelli and Colwell 2010). We chose these estimators because our data was incidence-based and because they outperform other estimators in terms of error and accuracy (Gotelli and Colwell 2010). We considered each visit to the area as a sampling unit and used the incidence of each species in our full sample of 22 visits to estimate richness. We ran analyses in EstimateS 9.1.o (Colwell 2013).

\section{RESULTS \\ Species richness}

We listed 211 species of birds for the RBMG region, 205 of which were recorded by us in the field within the limits of the reserve (Table 1 ). We detected Rhea americana (Linnaeus, 1758), Porphyrio martinicus (Linnaeus, 1766), Anthus furcatus d'Orbigny \& Lafresnaye, 1837 and Sporophila ruficollis Cabanis, 1851 solely in the vicinities of the reserve. Fulica armillata Vieillot, 1817 and Asio clamator (Vieillot, 1808) were not detected by us in the field, but were included based on specimens collected in the vicinities of the reserve. We provided documentation for 186 species, including voice recordings and specimens collected by Willian Belton in the 1970 s and specimens collected by Emil Kaempfer in 1931 (Table 1).

Species richness was estimated at $223.83(\mathrm{SD}=3.88)$ and $214.68(S D=4.71)$ species using the Jackknife 2 and Chao 2 estimators, respectively. Thus, observed richness corresponds to $93.82 \%$ and $97.82 \%$ of the estimated richness.

\section{Distributional novelties}

Records of the following species constitute distributional novelties within Rio Grande do Sul: Sarkidiornis sylvicola Ihering \& Ihering, 1907, Tigrisoma lineatum (Boddaert, 1783), Laterallus melanophaius (Vieillot, 1819), Pardirallus maculatus (Boddaert, 1783), Chroicocephalus cirrocephalus (Vieillot, 1818), Micrococcyx cinereus (Vieillot, 1817), Bubo virginianus (Gmelin, 1788), Melanerpes candidus (Otto, 1796), Euscarthmus meloryphus Wied, 1831, Elaenia flavogaster (Thunberg, 1822), Icterus pyrrhopterus (Vieillot, 1819), Saltator similis d'Orbigny \& Lafresnaye, 1837, S. ruficollis, Sporophila cinnamomea (Lafresnaye, 1839), and Euphonia chlorotica (Linnaeus, 1766). Records of poorly known species in Rio Grande do Sul are detailed below, with the exception of S. ruficollis 
Table 1. List of bird species recorded in the Reserva Biológica do Mato Grande, state of Rio Grande do Sul, southern Brazil, with conservation status, endemism and voucher details. Conservation status in state (RS, sensu Rio Grande do Sul 2014), national (Br, sensu MMA 2014) and global (Gl, sensu IUCN 2014) levels: EN - Endangered, VU - Vulnerable, and NT - Near-threatened. Endemism (sensu Bencke et al. 2006): Pampas - Pa, and Atlantic Forest - AF. Museum acronyms: AMNH - American Museum of Natural History, New York, USA, MCN - Museu de Ciências Naturais, Fundação Zoobotânica do Rio Grande do Sul, Porto Alegre, Brazil.

\begin{tabular}{|c|c|c|c|c|}
\hline & Status & Photo & Voice & Specimen \\
\hline \multicolumn{5}{|l|}{ RHEIFORMES Forbes, 1884} \\
\hline \multicolumn{5}{|l|}{ Rheidae Bonaparte, 1849} \\
\hline Rhea americana (Linnaeus, 1758) & NT (Gl) & & & AMNH321725 \\
\hline \multicolumn{5}{|l|}{ TINAMIFORMES Huxley, 1872} \\
\hline \multicolumn{5}{|l|}{ Tinamidae Gray, 1840} \\
\hline Nothura maculosa (Temminck, 1815) & & wikiaves.com/1497830 & & \\
\hline \multicolumn{5}{|l|}{ ANSERIFORMES Linnaeus, 1758} \\
\hline \multicolumn{5}{|l|}{ Anhimidae Stejneger, 1885} \\
\hline Chauna torquata (Oken, 1816) & & wikiaves.com/1502834 & macaulaylibrary.org/audio/18847 & AMNH321726 \\
\hline \multicolumn{5}{|l|}{ Anatidae Leach, 1820} \\
\hline Dendrocygna bicolor (Vieillot, 1816) & & wikiaves.com/1115552 & & \\
\hline Dendrocygna viduata (Linnaeus, 1766) & & wikiaves.com/1503468 & & \\
\hline Cygnus melancoryphus (Molina, 1782) & & wikiaves.com/1500346 & & \\
\hline Coscoroba coscoroba (Molina, 1782) & & wikiaves.com/1118366 & & \\
\hline Sarkidiornis sylvicola Ihering \& Ihering, 1907 & & wikiaves.com/1150786 & & \\
\hline Callonetta leucophrys (Vieillot, 1816) & & wikiaves.com/1138368 & & \\
\hline Amazonetta brasiliensis (Gmelin, 1789) & & wikiaves.com/1135087 & & \\
\hline Anas flavirostris Vieillot, 1816 & & & xeno-canto.org/22557 & \\
\hline Anas georgica Gmelin, 1789 & & & xeno-canto.org/20597 & \\
\hline Anas versicolor Vieillot, 1816 & & wikiaves.com/1496641 & & \\
\hline Netta peposaca (Vieillot, 1816) & & & xeno-canto.org/23277 & \\
\hline
\end{tabular}

\section{PODICIPEDIFORMES Fürbringer, 1888}

Podicipedidae Bonaparte, 1831

Rollandia rolland (Quoy \& Gaimard, 1824) $\quad$ wikiaves.com/1019623

Podilymbus podiceps (Linnaeus, 1758) W wikiaves.com/1118758

\begin{tabular}{ll} 
Podicephorus major (Boddaert, 1783) $\quad$ wikiaves.com $/ 1503473$ \\
\hline
\end{tabular}

PHOENICOPTERIFORMES Fürbringer, 1888

Phoenicopteridae Bonaparte, 1831

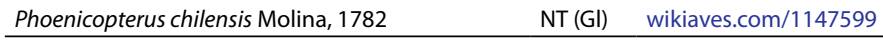

CICONIIFORMES Bonaparte, 1854

Ciconiidae Sundevall, 1836

Ciconia maguari (Gmelin, 1789) wikiaves.com/1139326

Mycteria americana Linnaeus, $1758 \quad$ wikiaves.com/1115548

\section{SULIFORMES Sharpe, 1891}

Phalacrocoracidae Reichenbach, 1849

Phalacrocorax brasilianus (Gmelin, 1789)

\section{PELECANIFORMES Sharpe, 1891}

Ardeidae Leach, 1820

Tigrisoma lineatum (Boddaert, 1783)

Botaurus pinnatus (Wagler, 1829)

Nycticorax nycticorax (Linnaeus, 1758)

Butorides striata (Linnaeus, 1758)

Bubulcus ibis (Linnaeus, 1758)

Ardea cocoi Linnaeus, 1766

Ardea alba Linnaeus, 1758

Syrigma sibilatrix (Temminck, 1824)

Egretta thula (Molina, 1782)

Threskiornithidae Poche, 1904

Plegadis chihi (Vieillot, 1817)

wikiaves.com/1509170

AMNH321801

Phimosus infuscatus (Lichtenstein, 1823)

wikiaves.com/1497891

wikiaves.com/1137437

AMNH321789; AMNH321790; AMNH321791

wikiaves.com/1137436

wikiaves.com/1137433

wikiaves.com/1136405

wikiaves.com /1497926

wikiaves.com/1506344

Theristicus caerulescens (Vieillot, 1817)

wikiaves.com/1115555

MCN366; MCN369

wikiaves.com/1147598

AMNH321775; AMNH321776; AMNH321777;

Platalea ajaja Linnaeus, 1758

wikiaves.com/1153857

AMNH321778; AMNH321779

\section{CATHARTIFORMES Seebohm, 1890}

Cathartidae Lafresnaye, 1839

Cathartes aura (Linnaeus, 1758)

Cathartes burrovianus Cassin, 1845 
Table 1. Continued.

\begin{tabular}{|c|c|c|c|c|}
\hline & Status & Photo & Voice & Specimen \\
\hline \multicolumn{5}{|l|}{ ACCIPITRIFORMES Bonaparte, 1831} \\
\hline \multicolumn{5}{|l|}{ Accipitridae Vigors, 1824} \\
\hline Circus cinereus Vieillot, 1816 & $\begin{array}{l}\mathrm{VU} \\
(\mathrm{Br}, \mathrm{RS})\end{array}$ & $\begin{array}{l}\text { wikiaves.com/1181956; } \\
\text { wikiaves.com/1503464 }\end{array}$ & & \\
\hline Circus buffoni (Gmelin, 1788) & & wikiaves.com/1506345 & & \\
\hline Rostrhamus sociabilis (Vieillot, 1817) & & wikiaves.com/1147602 & & \\
\hline Heterospizias meridionalis (Latham, 1790) & & wikiaves.com/1141185 & & \\
\hline Urubitinga urubitinga (Gmelin, 1788) & & wikiaves.com/733901 & & \\
\hline Rupornis magnirostris (Gmelin, 1788) & & wikiaves.com/1496628 & & \\
\hline \multicolumn{5}{|l|}{ GRUIFORMES Bonaparte, 1854} \\
\hline \multicolumn{5}{|l|}{ Aramidae Bonaparte, 1852} \\
\hline Aramus guarauna (Linnaeus, 1766) & & wikiaves.com/1136404 & & MCN676; AMNH321774; AMNH321774 \\
\hline \multicolumn{5}{|l|}{ Rallidae Rafinesque, 1815} \\
\hline Aramides ypecaha (Vieillot, 1819) & & wikiaves.com/1509174 & & \\
\hline Laterallus melanophaius (Vieillot, 1819) & & & xeno-canto.org/23276 & \\
\hline Pardirallus maculatus (Boddaert, 1783) & & wikiaves.com/1496634 & & \\
\hline Pardirallus sanguinolentus (Swainson, 1837) & & & xeno-canto.org/21154 & \\
\hline Gallinula galeata (Lichtenstein, 1818) & & wikiaves.com/1299241 & xeno-canto.org/22561 & MCN361 \\
\hline \multicolumn{5}{|l|}{ Gallinula melanops (Vieillot, 1819) } \\
\hline Porphyrio martinicus (Linnaeus, 1766) & & wikiaves.com /1491719 & & \\
\hline Fulica armillata Vieillot, 1817 & & & & MCN694 \\
\hline Fulica ruffrons Philippi \& Landbeck, 1861 & & wikiaves.com/1120863 & & \\
\hline Fulica leucoptera Vieillot, 1817 & & wikiaves.com/1134977 & & MCN697 \\
\hline
\end{tabular}

\section{CHARADRIIFORMES Huxley, 1867}

Charadriidae Leach, 1820

Vanellus chilensis (Molina, 1782)

wikiaves.com/1161338

AMNH321750

Pluvialis dominica (Statius Muller, 1776)

Charadrius semipalmatus Bonaparte, 1825

Charadrius collaris Vieillot, 1818

wikiaves.com/1149198

wikiaves.com/1113937

Charadrius modestus Lichtenstein, 1823

Haematopodidae Bonaparte, 1838

Haematopus palliatus Temminck, 1820

Recurvirostridae Bonaparte, 1831

Himantopus melanurus Vieillot, 1817

Scolopacidae Rafinesque, 1815

Gallinago paraguaiae (Vieillot, 1816)

Tringa solitaria Wilson, 1813

Tringa melanoleuca (Gmelin, 1789)

Tringa flavipes (Gmelin, 1789)

Calidris fuscicollis (Vieillot, 1819)

Calidris melanotos (Vieillot, 1819)

Calidris subruficollis (Vieillot, 1819)

Jacanidae Chenu \& Des Murs, 1854

Jacana jacana (Linnaeus, 1766)

Laridae Rafinesque, 1815

Chroicocephalus maculipennis (Lichtenstein, 1823)

Chroicocephalus cirrocephalus (Vieillot, 1818)

Larus dominicanus Lichtenstein, 1823

Sternidae Vigors, 1825

Sternula superciliaris (Vieillot, 1819)

Phaetusa simplex (Gmelin, 1789)

Gelochelidon nilotica (Gmelin, 1789)

Sterna trudeaui Audubon, 1838

Rynchopidae Bonaparte, 1838

Rynchops niger Linnaeus, 1758

Columbiformes Latham, 1790

Columbidae Leach, 1820

Columbina talpacoti (Temminck, 1811)

Columbina picui (Temminck, 1813)

Patagioenas picazuro (Temminck, 1813) wikiaves.com/1120882

wikiaves.com/1181955

wikiaves.com/1491717

wikiaves.com/1153859

wikiaves.com/1118362

wikiaves.com/1138367; wikiaves.com/1503499

NT (Gl), wikiaves.com/1644460

$\mathrm{VU}(\mathrm{Br})$

wikiaves.com/1141183

wikiaves.com/1122474

wikiaves.com/1142906

wikiaves.com/1115550

wikiaves.com/1115553

xeno-canto.org/22563

xeno-canto.org/21152

xeno-canto.org/20607

xeno-canto.org/22562
MCN720

xeno-canto.org/200410;

macaulaylibrary.org/audio/18877

macaulaylibrary.org/audio/18878

AMNH321759; AMNH321760; AMNH321761; AMNH321762; AMNH321763

AMNH321773

AMNH321745; AMNH321746; AMNH321747; AMNH321748; AMNH321749 wikiaves.com /1503483

wikiaves.com/1115549 
Table 1. Continued.

\begin{tabular}{|c|c|c|c|c|}
\hline & Status & Photo & Voice & Specimen \\
\hline Zenaida auriculata (Des Murs, 1847) & & wikiaves.com/1161340 & & \\
\hline Leptotila verreauxi Bonaparte, 1855 & & wikiaves.com/1508310 & xeno-canto.org/200395 & \\
\hline \multicolumn{5}{|l|}{ CUCULIFORMES Wagler, 1830} \\
\hline \multicolumn{5}{|l|}{ Cuculidae Leach, 1820} \\
\hline Micrococcyx cinereus (Vieillot, 1817) & & & xeno-canto.org/29035 & \\
\hline \multicolumn{5}{|l|}{ Piaya cayana (Linnaeus, 1766) } \\
\hline Coccyzus melacoryphus Vieillot, 1817 & & wikiaves.com/1139328 & macaulaylibrary.org/audio/19005 & \\
\hline Crotophaga ani Linnaeus, 1758 & & wikiaves.com/1500347 & & \\
\hline Guira guira (Gmelin, 1788) & & wikiaves.com/1141184 & & MCN835 \\
\hline \multicolumn{5}{|l|}{ STRIGIFORMES Wagler, 1830} \\
\hline \multicolumn{5}{|l|}{ Tytonidae Mathews, 1912} \\
\hline \multicolumn{5}{|l|}{ Tyto furcata (Temminck, 1827) } \\
\hline \multicolumn{5}{|l|}{ Strigidae Leach, 1820} \\
\hline Megascops choliba (Vieillot, 1817) & & & xeno-canto.org/22565 & MCN806 \\
\hline Bubo virginianus (Gmelin, 1788) & & & xeno-canto.org/23275 & \\
\hline Athene cunicularia (Molina, 1782) & & wikiaves.com/1137434 & & \\
\hline Asio clamator (Vieillot, 1808) & & & macaulaylibrary.org/audio/19012 & MCN817 \\
\hline
\end{tabular}

\section{CAPRIMULGIFORMES Ridgway, 1881}

Caprimulgidae Vigors, 1825

Hydropsalis torquata (Gmelin, 1789)

wikiaves.com/1141181

Chordeiles nacunda (Vieillot, 1817)

Chordeiles minor (Forster, 1771)

\section{APODIFORMES Peters, 1940}

Trochilidae Vigors, 1825

Chlorostilbon lucidus (Shaw, 1812)

wikiaves.com/1138371

Hylocharis chrysura (Shaw, 1812)

wikiaves.com/1141182

\section{CORACIIFORMES Forbes, 1844}

Alcedinidae Rafinesque, 1815

Megaceryle torquata (Linnaeus, 1766)

Chloroceryle amazona (Latham, 1790)

Chloroceryle americana (Gmelin, 1788)

wikiaves.com /1497824

wikiaves.com/1118761

wikiaves.com/1505170

AMNH321814; AMNH321816

PICIFORMES Meyer \& Wolf, 1810

Picidae Leach, 1820

Melanerpes candidus (Otto, 1796)

Veniliornis spilogaster (Wagler, 1827)

Colaptes melanochloros (Gmelin, 1788)

$\mathrm{AF}$

Colaptes campestris (Vieillot, 1818)

FALCONIFORMES Bonaparte, 1831

Falconidae Leach, 1820

Caracara plancus (Miller, 1777)

Milvago chimachima (Vieillot, 1816)

Milvago chimango (Vieillot, 1816)

Falco sparverius Linnaeus, 1758

PSITTACIFORMES Wagler, 1830

Psittacidae Rafinesque, 1815

Myiopsitta monachus (Boddaert, 1783)

PASSERIFORMES Linnaeus, 1758

Thamnophilidae Swainson, 1824

Thamnophilus ruficapillus Vieillot, 1816

Thamnophilus caerulescens Vieillot, 1816

Furnariidae Gray, 1840

Cinclodes fuscus (Vieillot, 1818)

Furnarius rufus (Gmelin, 1788)

Limnornis curvirostris Gould, 1839

Phleocryptes melanops (Vieillot, 1817)

$\mathrm{Pa}$

wikiaves.com/1140290

wikiaves.com/1118376

Syndactyla rufosuperciliata (Lafresnaye, 1832)

Spartonoica maluroides (d'Orbigny \& Lafresnaye, 1837) wikiaves.com/1139330

wikiaves.com/1139329

wikiaves.com/1498373 xeno-canto.org/183674

wikiaves.com/265682

wikiaves.com/1504075

xeno-canto.org/200405

wikiaves.com/1503964

wikiaves.com/1151187

xeno-canto.org/20602

macaulaylibrary.org/audio/19235

macaulaylibrary.org/audio/19210; macaulaylibrary.org/audio/19234

xeno-canto.org/23278;

macaulaylibrary.org/audio/19236; macaulaylibrary.org/audio/19237; macaulaylibrary.org/audio/19238 
Table 1. Continued.

Anumbius annumbi (Vieillot, 1817)
Schoeniophylax phryganophilus (Vieillot, 1817)
Certhiaxis cinnamomeus (Gmelin, 1788)
Synallaxis frontalis Pelzeln, 1859
Synallaxis spixi Sclater, 1856
Limnoctites rectirostris (Gould, 1839)
Cranioleuca sulphurifera (Burmeister, 1869)

Cranioleuca sp.

Tityridae Gray, 1840

Pachyramphus polychopterus (Vieillot, 1818)

Tachurididae Ohlson, Irestedt, Ericson \& Fjeldså,

$$
2013
$$

Tachuris rubrigastra (Vieillot, 1817)

Rhynchocyclidae Berlepsch, 1907

Phylloscartes ventralis (Temminck, 1824)

Poecilotriccus plumbeiceps (Lafresnaye, 1846)

Tyrannidae Vigors, 1825

Euscarthmus meloryphus Wied, 1831

Camptostoma obsoletum (Temminck, 1824)

Elaenia flavogaster (Thunberg, 1822)

Elaenia parvirostris Pelzeln, 1868

Elaenia obscura (d'Orbigny \& Lafresnaye, 1837)

Pseudocolopteryx sclateri (Oustalet, 1892)

Pseudocolopteryx flaviventris (d'Orbigny \& Lafresnaye, 1837)

Serpophaga nigricans (Vieillot, 1817)

Serpophaga subcristata (Vieillot, 1817)

Myiarchus swainsoni Cabanis \& Heine, 1859

Pitangus sulphuratus (Linnaeus, 1766)

Machetornis rixosa (Vieillot, 1819)

Tyrannus melancholicus Vieillot, 1819

Tyrannus savana Vieillot, 1808

Myiophobus fasciatus (Statius Muller, 1776)

Pyrocephalus rubinus (Boddaert, 1783)

Arundinicola leucocephala (Linnaeus, 1764)

Lathrotriccus euleri (Cabanis, 1868)

Lessonia rufa (Gmelin, 1789)

Knipolegus cyanirostris (Vieillot, 1818)

Hymenops perspicillatus (Gmelin, 1789)

Satrapa icterophrys (Vieillot, 1818)

Xolmis irupero (Vieillot, 1823)

Vireonidae Swainson, 1837

Cyclarhis gujanensis (Gmelin, 1789)

Hirundinidae Rafinesque, 1815

Pygochelidon cyanoleuca (Vieillot, 1817)

Alopochelidon fucata (Temminck, 1822)

Stelgidopteryx ruficollis (Vieillot, 1817)

Progne tapera (Vieillot, 1817)

Progne chalybea (Gmelin, 1789)

Tachycineta leucorrhoa (Vieillot, 1817)

Tachycineta leucopyga (Meyen, 1834)

Hirundo rustica Linnaeus, 1758

Troglodytidae Swainson, 1831

Troglodytes musculus Naumann, 1823

Polioptilidae Baird, 1858

Polioptila dumicola (Vieillot, 1817)

Turdidae Rafinesque, 1815

Turdus rufiventris Vieillot, 1818

Turdus amaurochalinus Cabanis, 1850

Turdus albicollis Vieillot, 1818

\section{Status Photo}

wikiaves.com/733922

Wikiaves.com/733485

NT (Gl) wikiaves.com/1135218

$\mathrm{Pa} \quad$ wikiaves.com/1120851

wikiaves.com/734915; wikiaves.com/1181965

wikiaves.com/1146752

wikiaves.com/1120874

wikiaves.com/737964

$$
\text { xeno-canto.org/200406 }
$$

xeno-canto.org/200393

xeno-canto.org/200401

xeno-canto.org/200394

xeno-canto.org/24003

wikiaves.com/1118378

xeno-canto.org/201260

wikiaves.com/1150788

wikiaves.com/738705

wikiaves.com/1504080

wikiaves.com/1142909

wikiaves.com/1153860

wikiaves.com/1161337

wikiaves.com/1181964

wikiaves.com/1505164

wikiaves.com/1118341

wikiaves.com/1142907

wikiaves.com/1462552

wikiaves.com/1299239

wikiaves.com/1504086

wikiaves.com/1150787

wikiaves.com/1508313

wikiaves.com/736928

xeno-canto.org/20609

xeno-canto.org/20600

wikiaves.com/1491779

wikiaves.com/1149201

wikiaves.com/1497928

wikiaves.com/1115544

wikiaves.com/1151186

wikiaves.com/1497823

wikiaves.com/1107567

wikiaves.com/737963

xeno-canto.org/21643

xeno-canto.org/200397

wikiaves.com/1496625

xeno-canto.org/21642

xeno-canto.org/200404

xeno-canto.org/21150
Specimen

AMNH321886

macaulaylibrary.org/audio/19539

AMNH321934; AMNH321935

AMNH321924; AMNH321925; AMNH321926

AMNH321911

MCN1004; AMNH321920; AMNH321921; AMNH321922; AMNH321923

MCN1009; AMNH321931; AMNH321932;

AMNH321933

AMNH321905; AMNH321906

AMNH322013

xeno-canto.org/200396 
Table 1. Continued.

\begin{tabular}{|c|c|c|c|c|}
\hline & Status & Photo & Voice & Specimen \\
\hline \multicolumn{5}{|l|}{ Mimidae Bonaparte, 1853} \\
\hline Mimus saturninus (Lichtenstein, 1823) & & wikiaves.com/1142910 & & \\
\hline Mimus triurus (Vieillot, 1818) & & wikiaves.com/1000886 & & \\
\hline \multicolumn{5}{|l|}{ Motacillidae Horsfield, 1821} \\
\hline Anthus lutescens Pucheran, 1855 & & & xeno-canto.org/200391 & \\
\hline Anthus furcatus d'Orbigny \& Lafresnaye, 1837 & & & xeno-canto.org/22564 & \\
\hline Anthus correndera Vieillot, 1818 & & wikiaves.com/1491766 & $\begin{array}{l}\text { xeno-canto.org/201254; } \\
\text { macaulaylibrary.org/audio/20134 }\end{array}$ & \\
\hline \multicolumn{5}{|l|}{ Passerellidae Cabanis \& Heine, 1850} \\
\hline Zonotrichia capensis (Statius Muller, 1776) & & & xeno-canto.org/200411 & \\
\hline Ammodramus humeralis (Bosc, 1792) & & & xeno-canto.org/200399 & \\
\hline \multicolumn{5}{|c|}{ Parulidae Wetmore, Friedmann, Lincoln, Miller, Peters, van Rossem, Van Tyne \& Zimmer 1947} \\
\hline Setophaga pitiayumi (Vieillot, 1817) & & & xeno-canto.org/200408 & \\
\hline Geothlypis aequinoctialis (Gmelin, 1789) & & wikiaves.com/1140292 & & \\
\hline Myiothlypis leucoblephara (Vieillot, 1817) & $\mathrm{AF}$ & & xeno-canto.org/200392 & \\
\hline \multicolumn{5}{|l|}{ Icteridae Vigors, 1825} \\
\hline Icterus pyrrhopterus (Vieillot, 1819) & & & xeno-canto.org/200402 & \\
\hline Amblyramphus holosericeus (Scopoli, 1786) & & wikiaves.com/1299222 & & AMNH322088 \\
\hline Agelasticus thilius (Molina, 1782) & & & xeno-canto.org/200398 & AMNH322102 \\
\hline Chrysomus ruficapillus (Vieillot, 1819) & & wikiaves.com/1121200 & & \\
\hline Pseudoleistes virescens (Vieillot, 1819) & & wikiaves.com/1149202 & & AMNH322081; AMNH322082 \\
\hline Agelaioides badius (Vieillot, 1819) & & wikiaves.com/1135085 & & \\
\hline Molothrus rufoaxillaris Cassin, 1866 & & wikiaves.com/1497934 & & \\
\hline Molothrus bonariensis (Gmelin, 1789) & & wikiaves.com/1503970 & & \\
\hline Sturnella superciliaris (Bonaparte, 1850) & & wikiaves.com/1151185 & & AMNH322092 \\
\hline \multicolumn{5}{|l|}{ Thraupidae Cabanis, 1847} \\
\hline \multicolumn{5}{|l|}{ Coereba flaveola (Linnaeus, 1758) } \\
\hline Saltator similis d'Orbigny \& Lafresnaye, 1837 & & wikiaves.com/1503501 & xeno-canto.org/201257 & \\
\hline Saltator aurantiirostris Vieillot, 1817 & & wikiaves.com/1150785 & xeno-canto.org/20611 & \\
\hline \multicolumn{5}{|l|}{ Tangara preciosa (Cabanis, 1850) } \\
\hline Stephanophorus diadematus (Temminck, 1823) & & wikiaves.com/1495885 & & \\
\hline Paroaria coronata (Miller, 1776) & & wikiaves.com/996688 & & \\
\hline Pipraeidea bonariensis (Gmelin, 1789) & & wikiaves.com/1498404 & xeno-canto.org/200407 & \\
\hline Donacospiza albifrons (Vieillot, 1817) & & wikiaves.com/1113931 & xeno-canto.org/201259 & \\
\hline Poospiza nigrorufa (d'Orbigny \& Lafresnaye, 1837) & & wikiaves.com/1299226 & $\begin{array}{l}\text { xeno-canto.org/20608; } \\
\text { macaulaylibrary.org/audio/19322 }\end{array}$ & AMNH322049; AMNH322050; AMNH322051 \\
\hline Sicalis flaveola (Linnaeus, 1766) & & wikiaves.com/1504081 & & AMNH322052 \\
\hline Sicalis luteola (Sparrman, 1789) & & wikiaves.com/1151184 & & \\
\hline Embernagra platensis (Gmelin, 1789) & & wikiaves.com/1508315 & & \\
\hline Volatinia jacarina (Linnaeus, 1766) & & wikiaves.com/1161339 & & \\
\hline Sporophila collaris (Boddaert, 1783) & & wikiaves.com/1107578 & & MCN1420 \\
\hline Sporophila caerulescens (Vieillot, 1823) & & & $\begin{array}{l}\text { xeno-canto.org/200409; } \\
\text { macaulaylibrary.org/audio/20262 }\end{array}$ & \\
\hline Sporophila ruficollis Cabanis, 1851 & $\begin{array}{l}\mathrm{VU} \\
\text { (RS, Br) }\end{array}$ & $\begin{array}{l}\text { wikiaves.com } / 451759 \\
\text { wikiaves.com } / 1616273\end{array}$ & & \\
\hline Sporophila palustris (Barrows, 1883) & $\begin{array}{l}\text { EN (GI); } \\
\text { VU (RS, } \\
\mathrm{Br}) ; \mathrm{Pa}\end{array}$ & wikiaves.com/455703 & & \\
\hline Sporophila cinnamomea (Lafresnaye, 1839) & $\begin{array}{l}\mathrm{VU}(\mathrm{Gl}) \\
\mathrm{Pa}\end{array}$ & wikiaves.com/455683 & & \\
\hline \multicolumn{5}{|l|}{ Cardinalidae Ridgway, 1901} \\
\hline $\begin{array}{l}\text { Cyanoloxia glaucocaerulea (d'Orbigny \& } \\
\text { Lafresnaye, 1837) }\end{array}$ & & & xeno-canto.org/200400 & \\
\hline Cyanoloxia brissonii (Lichtenstein, 1823) & & wikiaves.com/1140287 & xeno-canto.org/201256 & \\
\hline \multicolumn{5}{|l|}{ Fringillidae Leach, 1820} \\
\hline Sporagra magellanica (Vieillot, 1805) & & wikiaves.com/1501963 & & \\
\hline \multicolumn{5}{|l|}{ Euphonia chlorotica (Linnaeus, 1766) } \\
\hline \multicolumn{5}{|l|}{ Passeridae Rafinesque, 1815} \\
\hline
\end{tabular}


and S. cinnamomea, dealt under the "threatened species" section.

Sarkidiornis sylvicola. Four individuals in female plumage were observed standing in a flooded grassy marsh with nearly 500 Dendrocygna viduata (Linnaeus, 1766) on 21 June 2008.

Pardirallus maculatus. A single individual was observed in a wet roadside with grassy vegetation on 14 September 2008 and in a patch with similar conditions amidst Eryngium pandanifolium at the edge of the marsh on 23 October 2014.

Chroicocephalus cirrocephalus. A few individuals were observed along the Lagoa Mirim, usually with Chroicocephalus maculipennis (Lichtenstein, 1823), on 14 field trips covering all seasons of the year.

Micrococcyx cinereus. One to two individuals were recorded in small patches of restinga forest from 13 December 2008 to late January 2009.

Euscarthmus meloryphus. One individual was observed in restinga forest on 15-16 November 2008 and 13 December 2008.

Elaenia flavogaster. A pair observed in sandy restinga forest at the northern limit of the reserve on 17 July 2008 constitutes the only record for our study area.

\section{Endemic species}

We recorded five species endemic to the Pampas biogeographic province: Limnornis curvirostris Gould, 1839, Spartonoica maluroides (d'Orbigny \& Lafresnaye, 1837), Cranioleuca sulphurifera (Burmeister, 1869), Sporophila palustris (Barrows, 1883) and S. cinnamomea. Veniliornis spilogaster (Wagler, 1827) and Myiothlypis leucoblephara (Vieillot, 1817) were the only Atlantic Forest endemics recorded.

\section{Species of conservation concern}

Nine species are threatened or near-threatened with extinction in state (RS), national (BR) and/or global (GL) levels. Occurrence and abundance of these species is detailed below.

Rhea americana (GL - Near-threatened). Small groups of 1-5 individuals were frequently observed in grasslands and fallow rice fields that mark the northern limits of the reserve. This species was not recorded using habitats within the reserve.

Phoenicopterus chilensis Molina, 1782 (GL - Nearthreatened). A lone individual was observed foraging on the shores of the Lagoa Mirim on 24 January 2009.

Circus cinereus Vieillot, 1816 (RS, BR - Vulnerable). Lone individuals were frequently observed hunting in low flight over grasslands and wetlands throughout the area, especially during the winter. Although we did not observe evidences of breeding within the reserve, the RBMG constitutes an important foraging ground for this harrier.

Calidris subruficollis (Vieillot, 1819) (BR - Vulnerable;
GL - Near-threatened). We observed eight individuals standing on the beach at the Lagoa Mirim on 22 February 2008 , and seven birds in an overgrazed grassland on the outskirts of the reserve on 24 October 2008.

Spartonoica maluroides (GL - Near-threatened). As much as 25 individuals were recorded per day in tall emergent macrophytes and grasses during the autumn and winter. Rarer during the spring and summer. Presence of young birds and records of territorial adults during this period suggests local breeding.

Limnoctites rectirostris (Gould, 1839) (GL - Nearthreatened). Occurs in E. pandanifolium patches that mark the transition between wetlands and higher terrain to the north and also in patches of this plant that occur in seasonally flooded grasslands along the Canal São Gonçalo (Fig. 3 d, e). We estimate that approximately 15 pairs occur within the RBMG.

Sporophila ruficollis (RS, BR - Vulnerable; GL - Nearthreatened). A male with plumage corresponding do the type morph (grey cap, chocolate throat, pale creamy underparts and brownish upperparts; Areta et al. 2011) accompanied by a female-plumaged individual were observed on a roadside $\left(32^{\circ} 07^{\prime} 25^{\prime \prime} \mathrm{S}, 052^{\circ} 35^{\prime} 28^{\prime \prime} \mathrm{W}\right)$ across Santa Isabel in neighboring Rio Grande municipality on 6 and 7 February 2009. Birds were frequently observed feeding together on Paspalum urvillei (Poaceae) seeds. Despite intensive surveys for Sporophila seedeaters within the limits of the RBMG, we never recorded this species in the reserve.

Sporophila palustris (RS, BR - Vulnerable; GL - Endangered). Recorded on all expeditions between December 2008 and March 2009. This species is restricted to seasonally flooded wet grasslands that occur on the eastern border of the marsh along the Canal São Gonçalo (Figure $3 \mathrm{~d}$ ). Approximately seven pairs occur in the reserve. Two nests and adults feeding nestlings and fledglings were observed. Details of these breeding records and of the breeding biology of the species were described in Vizentin-Bugoni et al. (2013).

Sporophila cinnamomea (GL - Vulnerable). A lone male was observed on the eastern sector of the reserve on 13 and 19 December 2008. The same individual was presumably detected on both occasions.

\section{Roosts}

Six species roosted in large or moderate numbers in the reserve: Nycticorax nycticorax (Linnaeus, 1758), Ardea alba Linnaeus, 1758, Plegadis chihi (Vieillot, 1817), Rostrhamus sociabilis (Vieillot, 1817), Tachycineta leucorrhoa (Vieillot, 1817) and Chrysomus ruficapillus (Vieillot, 1819) (Table 2). Largest numbers corresponded to $P$. chihi (5,000 individuals) and C. ruficapillus (4,000 individuals). Roosts were located in stands of tall emergent macrophytes and sarandi bushes in the marsh located in the central sector of the RBMG. 
Table 2. Species, date and number of individuals congregating in roosts within the limits of the Reserva Biológica do Mato Grande, Rio Grande do Sul, Brazil.

\begin{tabular}{llc}
\hline Species & Date & Number of individuals \\
\hline Plegadis chihi & 15 March 2008 & 3,500 \\
& 19 April 2008 & 5,000 \\
& 21 June 2008 & 5,000 \\
& 23 August 2008 & 1,100 \\
& 24 October 2008 & 2,330 \\
& 25 March 2009 & 8,795 \\
Chrysomus ruficapillus & 17 May 2008 & 4,000 \\
Tachycineta leucorrhoa & 19 April 2008 & 600 \\
Ardea alba & 10 February 2009 & 90 \\
Rostrhamus sociabilis & 15 March 2008 & 80 \\
Nycticorax nycticorax & 24 October 2008 & 53 \\
\hline
\end{tabular}

\section{DISCUSSION \\ Species list}

The 211 species listed for the RBMG and adjacencies corresponds to almost one-third (31.9\%) of the total number of birds included in the primary list of the state of Rio Grande do Sul ( $n=661$; Bencke et al. 2010). Species richness is also high when compared to other wetlands in the state (Table 3). Direct comparisons of the total number of species recorded in these areas are hampered by a series of factors. Differences in species richness may relate to differences in sampling methods, especially temporal coverage, night sampling and use of playback to detect secretive species. There are also differences in the total area sampled and in habitats included in each study. Areas with the highest number of species (e.g., Accordi and Barcellos 2006) are larger and include various types of forest and grassland habitats adjacent to wetlands. Not surprisingly, species responsible for the differences in richness between the above mentioned localities and the RBMG are usually forest and grassland taxa.

Species included in our list but not recorded in the field (F. armillata and A. clamator) were collected by William Belton in the vicinities of the reserve in 1972 and 1974, respectively. Asio clamator is uncommon in the region (Belton 1994), and may have been overlooked during our surveys. Fulica armillata, on the other hand, is common in open marshes, reservoirs and lagoons in southern Rio Grande do Sul (Belton 1994), and was frequently recorded in the Lagoa Mirim across the RBMG

Table 3. Number of species recorded in wetlands in Rio Grande do Sul, Brazil.

\begin{tabular}{lll}
\hline Species & Locality & Source \\
\hline 211 & Reserva Biológica do Mato Grande & This study \\
220 & Estação Ecológica do Taim & Mähler-Jr. et al. (1996) \\
176 & Saco da Mangueira & Dias and Maurício (1998) \\
210 & Banhado dos Pachecos & Accordi and Hartz (2006) \\
283 & Catchment of the Lago Guaíba & Accordi and Barcellos (2006) \\
171 & Lagoa do Casamento & Bencke et al. (2007) \\
170 & Butiazais de Tapes & Bencke et al. (2007) \\
230 & Parque Nacional da Lagoa do Peixe & Harrison et al. (2013) \\
\hline
\end{tabular}

in neighboring Rio Grande municipality, sometimes in large numbers (RAD, pers. obs.). This coot is highly vagile and prone to local concentration (Belton 1994), and therefore easily overlooked. Unidentified coots observed at a distance on March 2008 could potentially be this species.

We detected in the field all species collected by Emil Kaempfer in Santa Isabel in the 1930s, which suggests that local extinctions were rare or did not occur in our study area. The only species Kaempfer collected near the reserve (some $15 \mathrm{~km}$ to the southeast) and that has been considered locally extinct is Gubernatrix cristata (Vieillot, 1817) (Bencke et al. 2003). However, based on information in Bencke et al. (2003), it is unlikely that suitable habitat for this species occurred in the reserve.

Estimates of species richness indicate that sampling effort was sufficient to detect most species present in the RBMG region. According to distributional information in Belton (1994), Mähler-Jr. et al. (1996), Maurício and Dias (1996, 2000), Dias and Maurício (1998) and Bencke et al. (2003), at least 27 additional species occur in our study area and may eventually be recorded in the reserve (Table 4 ). Considering information on habitat use, seasonal occurrence and abundance of these species in southern Rio Grande do Sul (Belton 1994; Mähler-Jr. et al. 1996; Maurício and Dias 1996, 2000), we believe that Ixobrychus involucris (Vieillot, 1823) and Nycticryphes semicollaris (Vieillot, 1816) are likely to occur within the reserve and were probably missed during fieldwork due to their inconspicuous behavior. Adequate habitat for Riparia riparia (Linnaeus, 1758) also occurs within the reserve. In fact, we may have observed this species on a few occasions during the summer, but since birds were always distant and could not be safely separated from overall similar young Pygochelidon cyanoleuca (Vieillot, 1817), we refrained from including the species in our list. Habitat within the reserve is also suitable for Anas platalea Vieillot, 1816, Heteronetta atricapilla (Merrem, 1841), Calidris canutus (Linnaeus, 1758), Calidris himantopus (Bonaparte, 1826) and Phalaropus tricolor (Vieillot, 1819). We may have failed to detect these species during fieldwork because of their local scarceness and seasonal occurrence in the region (Belton 1994; Maurício and Dias 1996).

We were able to document $88.2 \%$ of the species listed for the RBMG and surroundings. All species lacking documentation occur throughout southern Rio Grande do Sul and have been photographed and/or had their voices recorded in nearby areas (see www.wikiaves.com and www.xeno-canto.org).

Records for E. meloryphus in the RBMG are the first published for the southern coastal plain of Rio Grande do Sul and extend its known distribution approximately $300 \mathrm{~km}$ to the west based on the distributional maps available in Belton (1994), Ridgely and Tudor (1994) and 
Table 4. Species mentioned for the region of the Reserva Biológica do Mato Grande, Brazil, in Belton (1994), Mähler-Jr. et al. (1996), Maurício and Dias (1996, 2000) Dias and Maurício (1998) and Bencke et al. (2003), but not detected during field trips.

\begin{tabular}{l}
\hline ANSERIFORMES Linnaeus, 1758 \\
Anatidae Leach, 1820 \\
Anas sibilatrix Poeppig, 1829 \\
Anas cyanoptera Vieillot, 1816 \\
Anas platalea Vieillot, 1816 \\
Heteronetta atricapilla (Merrem, 1841) \\
Oxyura vittata (Philippi, 1860) \\
\hline PELECANIFORMES Sharpe, 1891 \\
Ardeidae Leach, 1820 \\
Ixobrychus involucris (Vieillot, 1823) \\
\hline CATHARTIFORMES Seebohm, 1890 \\
Cathartidae Lafresnaye, 1839 \\
Coragyps atratus (Bechstein, 1793) \\
\hline ACCIPITRIFORMES Bonaparte, 1831 \\
Accipitridae Vigors, 1824 \\
Elanus leucurus (Vieillot, 1818) \\
Geranoaetus albicaudatus (Vieillot, 1816) \\
Buteo swainsoni Bonaparte, 1838
\end{tabular}

\begin{tabular}{l}
\hline GRUIFORMES Bonaparte, 1854 \\
Rallidae Rafinesque, 1815 \\
Aramides cajaneus (Statius Muller, 1776) \\
Laterallus leucopyrrhus (Vieillot, 1819) \\
\hline CHARADRIIFORMES Huxley, 1867 \\
Charadriidae Leach, 1820 \\
Oreopholus ruficollis (Wagler, 1829) \\
Scolopacidae Rafinesque, 1815 \\
Limosa haemastica (Linnaeus, 1758) \\
Calidris canutus (Linnaeus, 1758) \\
Calidris himantopus (Bonaparte, 1826) \\
Phalaropus tricolor (Vieillot, 1819) \\
Rostratulidae Mathews, 1914 \\
Nycticryphes semicollaris (Vieillot, 1816) \\
\hline CUCULIFORMES Wagler, 1830 \\
Cuculidae Leach, 1820 \\
Tapera naevia (Linnaeus, 1766)
\end{tabular}

\author{
STRIGIFORMES Wagler, 1830 \\ Strigidae Leach, 1820 \\ Asio flammeus (Pontoppidan, 1763) \\ PASSERIFORMES Linnaeus, 1758 \\ Scleruridae Swainson, 1827 \\ Geositta cunicularia (Vieillot, 1816) \\ Tyrannidae Vigors, 1825 \\ Myiodynastes maculatus (Statius Muller, 1776) \\ Xolmis cinereus (Vieillot, 1816) \\ Vireonidae Swainson, 1837 \\ Vireo chivi (Vieillot, 1817) \\ Hirundinidae Rafinesque, 1815 \\ Riparia riparia (Linnaeus, 1758) \\ Motacillidae Horsfield, 1821 \\ Anthus hellmayri Hartert, 1909 \\ Thraupidae Cabanis, 1847 \\ Pipraeidea melanonota (Vieillot, 1819)
}

BirdLife International (2014). This species was recorded in the 1970s in western Rio Grande do Sul and in contiguous regions of Uruguay in the departments of Artigas and Salto (Gore and Gepp 1978; Belton 1994). Recent distributional information on the birds of Uruguay (Azpiroz 2001, 2012; Rocha 2008; Olmos 2009) map its presence for all departments along the Rio Uruguay as well as in San José, Florida and Canelones. The species has also been recently recorded in eastern Rio Grande do Sul from the Lago Guaíba catchment area (Accordi and Barcellos 2006) north to Capão da Canoa, and in the southwestern sector of the state along the Uruguayan border (Azpiroz 2012; www.wikiaves.com). Taken together, these observations suggest that the species is expanding its range to the south and east in Rio Grande do Sul and Uruguay.

Records of S. cinnamomea and S. ruficollis are also the first for the coastal plain of Rio Grande do Sul based on distribution maps in Belton (1994), Bencke et al. (2003) and BirdLife International (2014). The nearest population of S. cinnamomea occurs in grasslands of the Serra do Sudeste hills, some $40 \mathrm{~km}$ to the west of our study area (RAD, pers. obs.), whereas established populations of $S$. ruficollis are found along the Rio Uruguay, nearly $500 \mathrm{~km}$ away (Bencke et al. 2003; Azpiroz 2012). In Rio Grande do Sul, both species occur in tall-grass grasslands in regions of gently undulated terrain, and were never recorded in large wetlands (Belton 1994; Bencke et al. 2003). Since our records for both species in the RBMG may relate to vagrant individuals, we recommend that surveys should be carried out in the Canal São Gonçalo region to solve the status of occurrence of these birds in coastal Rio Grande do Sul.

The remaining species listed as distributional novelties have been recently recorded throughout southern Rio Grande do Sul (www.wikiaves.com; www.xeno-canto. org). With the exception of S. sylvicola and M. cinereus, all are widespread and some appear to be common as inferred from data in the WikiAves and Xenocanto websites. Additional studies are necessary to determine if these records can be attributed to range extension or range expansion in the state (sensu Frey 2009; see also a discussion in Dias et al. 2010).

\section{Roosts}

Tall stands of dense emergent aquatic vegetation are important roosting places for some congregational birds. The highest numbers of birds roosting in the reserve were recorded from March to October, which corresponds to the non-breeding season of the local avifauna (Maurício et al. 2013), and when the water level in wetlands is higher. Despite the fact that large numbers of birds roost within the reserve, we found no evidences of colonial breeding of waterbirds in the area.

In a regional context, the RBMG is one of many other roosting areas existent in the Canal São Gonçalo floodplains (Bencke et al. 2006). Although we recorded high numbers of $P$. chihi roosting in the reserve, we counted nearly 14,000 birds flying over our study area and heading for dormitories located to the east, in Rio Grande municipality across the village of Santa Isabel on 19 April 2008. Identification and conservation of other roosting sites in the region is also necessary.

\section{Conservation}

The large number of species associated with aquatic environments recorded in the RBMG, especially those belonging to the Anatidae, Ardeidae, Threskiornithidae and Rallidae families, suggests that this protected area is important for the conservation of waterbirds in a regional context. However, to fully understand the importance of the RBMG for waterbird conservation, 
additional studies focusing on populational aspects of waterbirds using the reserve and other wetlands in southern Brazil are needed.

Of the nine conservation-concern species recorded in our study area, only four use the RBMG on a regular basis and/or have populations established within the reserve. The RBMG is especially important for the conservation of the endemic $S$. palustris, which breeds in the reserve (Vizentin-Bugoni et al. 2013). The RBMG is also important for the conservation of $C$. cinereus, which occurs regularly, albeit in small numbers, and the endemic $S$. maluroides, which apparently breeds in the reserve and can be common during the winter. Although the population of $L$. rectirostris within the reserve is not large, most E. pandanifolium marshes in the coastal plain have been converted into rice plantations and remaining patches are highly degraded (Bencke et al. 2003). Therefore, the RBMG may be important for this species at least in a local context. Since one of the main threats to these species is habitat modification resulting from excessive use of fire, overgrazing and trampling in tall-herbaceous habitats (Bencke et al. 2003; Vizentin-Bugoni et al. 2013), it is likely that the full implementation of the reserve and removal of cattle would benefit these birds. Control in access by fisherman, hunters and trappers would also be beneficial, especially for S. palustris, which is occasionally captured (Vizentin-Bugoni et al. 2013). We emphasize, however, that the complete exclusion of fire and grazing disturbance in grassy environments in protected areas may lead to habitat change and loss of grass-dependent species (Pillar and Vélez 2010). Studies assessing impacts of cattle removal and fire suppression upon grassy habitats and grassland-dependent species in the reserve are therefore required.

Although individuals of the near-threatened R. americana were not observed within the limits of the RBMG, this species was frequent in grasslands and fallow rice fields adjacent to the reserve. A slight expansion of the current limits of the RBMG to the north would protect comparatively drier grassland habitats used by this species and ensure that more areas of the reserve would remain free from the effects of floods. The most feasible areas for expansion under this scenario are sandy grasslands unsuitable for agriculture that occur on the northeastern border of the reserve. Development and implementation of sustainable cattle ranching and rice growing practices in the buffer zone surrounding the RBMG would further benefit $R$. americana. Rice fields in the region also hold large concentrations of shorebirds (Dias et al. 2014), and the development of sustainable farming may help raise the conservation value of private areas around the reserve.

The remaining species of conservation importance recorded in the RBMG were rare and usually represented by few individuals. Considering the known distribution and preferred habitats of these species in the state (Bencke et al. 2003), we can infer that their occurrence in the reserve is occasional and may be even related to vagrancy.

In short, we have demonstrated that the RBMG hosts a large number of bird species and that the reserve is an important area for wetland and grassland dependent species, some of which are endemic and threatened with extinction. Our findings not only emphasize the need to properly manage and conserve this protected area, but also support the indication of the region as an Important Bird Area (Bencke et al. 2006), and as an "area of extreme importance for the conservation, sustainable use and benefit sharing of the Brazilian biodiversity" (MMA 2007). Adequate conservation of birds and their habitats can only be met if the RBMG is fully implemented and effectively managed and protected. We hope that this study serves as an initial step to guide the implementation of the reserve.

\section{ACKNOWLEDGEMENTS}

We thank the NGO "Grupo Ecológico Amantes da Natureza" and the reserve manager, Luciano Soares, for encouraging this study and for their commitment to the implementation of the RBMG. Vinicius A. G. Bastazini, Camila F. Bosenbecker, Maraísa R. Braga, Eneko A. Floristan, Andros T. Gianuca, Paulo R. Post and Helena Venzke accompanied us in the field. Landowners Nabor Cunha and José Sopena and supervisors Valdemar, José and Francisco helped with housing and access to the RBMG. J.V.B. received CAPES-PDSE grant (processo: 99999.008012/2014-08) while working on the final versions of the manuscript. We thank Jesper Sonne for improving the English in the manuscript and one anonymous reviewer for critical comments on a previous version of this manuscript. Rafael Ritter, Cláudio Timm and Helena Venzke shared photographic records from the reserve and helped us document some species. Márcio Repenning, Carla S. Fontana, Ismael Franz and Juan I. Areta helped to identify Sporophila ruficollis. Glayson A. Bencke (MCN), Carla S. Fontana (MCP) and Paul Sweet (AMNH) assisted with information on specimens deposited in bird collections under their supervision. Cristiano Agra Iserhard assisted with use of richness estimators.

\section{LITERATURE CITED}

Accordi, I. A. and A. Barcellos. 2006. Composição da avifauna em oito áreas úmidas da Bacia Hidrográfica do Lago Guaíba, Rio Grande do Sul. Revista Brasileira de Ornitologia 14(2):101-115. http:// www4.museu-goeldi.br/revistabrornito/revista/index.php/ BJO/article/view/2402

Accordi, I.A. and S.M. Hartz. 2006. Distribuição espacial e sazonal da avifauna em uma área úmida costeira do sul do Brasil. Revista Brasileira de Ornitologia 14(2):117-135. http://www4.museu-goeldi. br/revistabrornito/revista/index.php/BJO/article/view/2403

Areta, J.I., J. I. Noriega, L. Pagano and I. Roesler. 2011. Unravelling 
the ecological radiation of the capuchinos: systematics of Darkthroated Seedeater Sporophila ruficollis and description of a new dark-collared form. Bulletin of the British Ornithologists' Club 131:4-23.

Azpiroz, A. B. 2001. Aves del Uruguay. Lista e introducción a su biología y conservación. Montevideo: Aves Uruguay - GUPECA. 106 pp.

Azpiroz, A. B. 2012. Aves de las pampas y campos de Argentina, Brasil y Uruguay. Nueva Helvecia: PRESSUR. 352 pp.

Barbier, E.B., M.C. Acreman and D. Knowler. 1997. Economic valuation of wetlands: a guide for policy makers and planners. Gland: Ramsar Convention. 127 pp. http://www.ramsar.org/ sites/default/files/documents/pdf/lib/lib_valuation_e.pdf

Belton, W. 1994. Aves do Rio Grande do Sul, distribuição e biologia. São Leopoldo: Editora Unisinos. 584 pp.

Bencke, G.A., C.S. Fontana, R.A. Dias, G.N. Maurício and J.K.F. Mähler-Jr. 2003. Aves; pp. 189-479, in: C.S. Fontana, G.A. Bencke and R.E. Reis (eds.). Livro vermelho da fauna ameaçada de extinção no Rio Grande do Sul. Porto Alegre: Edipucrs.

Bencke, G.A., G.N. Maurício, P.F. Develey and J.M. Goerck (orgs.). 2006. Áreas Prioritárias para a Conservação de aves no Brasil. Parte I-estados do domínio da Mata Atlântica. São Paulo: SAVE Brasil. 494 pp.

Bencke, G.A., M.I. Burger, J.C.P. Dotto, D.L. Guadagnin, T.O. Leite and J O. Menegheti. 2007. Aves; pp. 316-355, in: F.G. Becker, R.A. Ramos and L.A. Moura (orgs.). Biodiversidade. Regiões da Lagoa do Casamento e dos Butiazais de Tapes, planície costeira do Rio Grande do Sul. Brasília: Ministério do Meio Ambiente.

Bencke, G.A., R.A. Dias, L. Bugoni, C.E. Agne, C.S. Fontana, G.N. Maurício and D.B. Machado. 2010. Revisão e atualização da lista das aves do Rio Grande do Sul, Brasil. Iheringia, Série Zoologia 100(4): 519-556. http://www.scielo.br/pdf/isz/v10on4/22.pdf

BirdLife International. 2014. IUCN Red List for Birds. Accessed at http://www.birdlife.org, 1 November 2014.

CBRO (Comitê Brasileiro de Registros Ornitológicos). 2014. Lista das Aves do Brasil. 11 ${ }^{\mathrm{a}}$ edição. 1 Jan 2014. Accessed at http://www. cbro.org.br, 25 October 2014.

Claramunt, S. 2002. Variación geográfica en Cranioleuca pyrrhophia y el límite con Cranioleuca obsoleta (Furnariidae). Ornitología Neotropical 13:255-266. https://sora.unm.edu/sites/default/ files/journals/on/vo13no3/po255-p0266.pdf

Colwell, R.K. 2013. EstimateS: Statistical Estimation of Species Richness and Shared Species from Samples. Version 9. Accessed at http:// purl.oclc.org/estimates, 6 November 2014.

Dias, R.A. and G.N. Maurício. 1998. Lista preliminar da avifauna da extremidade sudoeste do saco da Mangueira e arredores, Rio Grande, Rio Grande do Sul. Atualidades Ornitológicas 86: 10-11.

Dias, R.A, A. Gianuca, J. Vizentin-Bugoni and M.A.A. Coimbra. 2010. New documented records for two bird species in southernmost Brazil, including the first mention of Agriornis murina for the country and comments on vagrancy. Revista Brasileira de Ornitologia 18(2):124-129. http://www4.museugoeldi.br/ revistabrornito/revista/index.php/BJO/article/viewFile/3909/ pdf_772

Dias, R.A., D.E. Blanco, A.P. Goijman and M.E. Zaccagnini. 2014. Density, habitat use, and opportunities for conservation of shorebirds in rice fields in southeastern South America. Condor 116: 384-393. doi: 10.1650/CONDOR-13-160.1

Estação Agroclimatológica de Pelotas. 2014. Normais climatológicas-mensal/anual. Accessed at http://www.cpact. embrapa.br/agromet/estacao/mensal.html, 6 November 2014.

EPA (Environmental Protection Agency). 2014. Wetlands. Accessed at http://water.epa.gov/type/wetlands, 6 November 2014.

Frey, J. K. 2009. Distinguishing range expansions from previously undocumented populations using background data from museum records. Diversity and Distributions 15(2): 183-187. doi: 10.1111/j.1472-4642.2008.00552.x

Furness, R.W. and J.J.D. Greenwood (eds.). 1993. Birds as monitors of environmental change. London: Chapman \& Hall. 356 pp.

Gore, M.E.J. and A.R.M. Gepp. 1978. Las aves del Uruguay. Montevideo: Mosca Hnos. 283 pp.

Gotelli, N.J. and R.K. Colwell. 2010. Estimating species richness; pp. 39-54 in: A.E. Magurran and B.J. McGill (eds.). Biological diversity: frontiers in measurement and assessment. Oxford: Oxford University Press.

Guadagnin, D.L., Â.S. Peter, L.F.C. Perello and L. Maltchick. 2005. Spatial and temporal patterns of waterbird assemblages in fragmented wetlands of southern Brazil. Waterbirds 28(3): 261272. doi: 10.1675/1524-4695(2005)028[0261:SATPOW]2.0.CO;2

Harrison, N.M., M.J. Whitehouse and L.A.S.P. Madureira. 2013. Observations of the under-described avifauna of the Mostardas Peninsula, Rio Grande do Sul, Brazil. Check List 9(2): 391-399. doi: 10.15560/9.2.391

IBGE (Instituto Brasileiro de Geografia e Estatística). 1986. Folha SH.22 Porto Alegre e parte das Folhas SH. 21 Uruguaiana e SI.22 Lagoa Mirim: geologia, geomorfologia, pedologia, vegetação, uso potencial da terra. (Levantamento de Recursos Naturais, 33). Rio de Janeiro: IBGE. 791 pp.

IUCN. 2014. IUCN Red List of threatened species. Version 2014.2. Accessed at http://www.iucnredlist.org, 6 November 2014.

Keddy, P. 200o. Wetland ecology: principles and conservation. Cambridge: Cambridge University Press. 614 pp.

Klein, A.H.F. 1998. Clima regional; pp. 5-7, in: U. Seeliger, C. Odebrecht and J.P. Castello (eds.). Os ecossistemas costeiro e marinho no extremo sul do Brasil. Rio Grande: Ecoscientia.

Lees, A.C., L.N. Naka, A. Aleixo, M. Cohn-Haft, V.D.Q. Piacentini, M.P.D. Santos, and L.F. Silveira. 2014. Conducting rigorous avian inventories: Amazonian case studies and a roadmap for improvement. Revista Brasileira de Ornitologia 22(2): 107-120. http://www4.museu-goeldi.br/revistabrornito/revista/index. php/BJO/article/viewFile/5705/pdf_4

Mähler-Jr., J.K.F, A. Kindel and E.A.I. Kindel. 1996. Lista comentada das espécies de aves da Estação Ecológica do Taim, Rio Grande do Sul, Brasil. Acta Biologica Leopoldensia 18: 69-103.

Maltby, E. 1991. Wetland management goals: wise use and conservation. Landscape and Urban Planning 20(1): 9-18. doi: 10.1016/0169-2046(91)90085-Z

Maltchik, L., E.S. Costa, C.G. Becker and A.E. Oliveira. 2003. Inventory of wetlands of Rio Grande do Sul (Brazil). Pesquisas: Botânica 53: 89-100. http://www.ablimno.org.br/acta/pdf/ acta_limnologica_contents1602E_files/Art4_16(2).pdf

Maurício, G.N., G.A. Bencke, M. Repenning, D.B. Machado, R.A. Dias and L. Bugoni. 2013. Review of the breeding status of birds in Rio Grande do Sul, Brazil. Iheringia, Série Zoologia 103: 163-184. doi: 10.1590/So073-47212013000200012

Maurício, G.N. and R.A. Dias. 1996. Novos registros e extensões de distribuição de aves palustres e costeiras no litoral sul do Rio Grande do Sul. Revista Brasileira de Ornitologia 4(1): 47-51. http://www4.museu-goeldi.br/revistabrornito/revista/index. php/BJO/article/viewFile/o410/pdf_87

Maurício, G.N. and R.A. Dias. 2000. New distributional information for birds in southern Rio Grande do Sul, Brazil, and the first record of the Rufous Gnateater Conopophaga lineata for Uruguay. Bulletin of the British Ornithologists' Club 120(4): 230-237. http://biodiversitylibrary.org/page/40025811

MMA (Ministério do Meio Ambiente) 2007. Áreas Prioritárias para a Conservação, uso sustentável e repartição dos recursos da biodiversidade Brasileira: Atualização - Portaria MMA n ${ }^{\circ} 9$ de 23 de Janeiro de 2007. Ministério do Meio Ambiente, Secretaria de Biodiversidade e Florestas. (Série Biodiversidade, 31). Brasília: MMA. $300 \mathrm{pp}$.

MMA (Ministério do Meio Ambiente). 2014. Lista nacional oficial de 
espécies da fauna ameaçadas de extinção - Portaria MMA n 444 de 17 de dezembro de 2014. Brasília. Diário Oficial da União: 245: 121-126.

Naumburg, E.M.B. 1935. Gazetteer and maps showing collecting stations visited by Emil Kaempfer in eastern Brazil and Paraguay. Bulletin of the American Museum of Natural History 68(6): 449-469. http://hdl.handle.net/2246/360

Olmos, A. 2009. Aves en el Uruguay. Montevideo: Trandinco. 520 pp.

Pillar, V.D.P. and E. Vélez. 2010. Extinção dos campos sulinos em unidades de conservação: um fenômeno natural ou um problema ético? Natureza \& Conservação 8(1): 84-86. doi: 10.4322/ natcon.00801014

Rocha, G. 2008. Aves del Uruguay. El país de los pájaros pintados. Volume III. Montevideo: Banda Oriental. 136 pp.

Rio Grande do Sul. 2014. Lista das espécies da fauna ameaçadas de extinção no estado do Rio Grande do Sul. Instrução Normativa n ${ }^{\circ}$ 3. DECRETO N ${ }^{\circ}$ 51.797. Porto Alegre, Diário Oficial do Estado do Rio Grande do Sul, Secretaria do Meio Ambiente, 8 de setembro de 2014.
Ridgely, R.S. and G. Tudor. 1994. The birds of South America. Volume II, the suboscine passerines. Austin: University of Texas Press. 940 pp. Vizentin-Bugoni J., J.I. Areta, A.G. Di Giacomo, A.S. Di Giacomo, F.P. Jacobs, M.A.A. Coimbra and R.A. Dias. 2013. Breeding biology and conservation of the Marsh Seedeater (Sporophila palustris). Bird Conservation International 23(2): 147-158. doi: 10.1017/ So959270913000221

Weller, M.W. 1999. Wetland birds: habitat resources and conservation implications. Cambridge: Cambridge University Press. 316 pp.

Authors' contribution statement: JVB and RAD conceived the investigation, collected and analysed the data, and wrote the text; FJ and MAAC collected the data.

Received: November 2014

Accepted: March 2015

Editorial responsibility: Boris Tinoco 